\title{
全球海洋预报与科学大数据
}

王辉“，刘娜，逢仁波，孙晓宇

国家海洋环境预报中心，北京 100081

* 联系人, E-mail: wangh@nmefc.gov.cn

2014-10-17 收稿, 2014-12-30 接受, 2015-01-20 网络版发表

国家科技支撑计划(2011BAC03B00)和国家自然科学基金(41106023)资助

摘要 全球海洋预报是当前国内外海洋预报领域的前沿方向之一，与实施海洋强国战略、维护 国家海洋权益, 以及开发深远海资源等各类海洋活动日益走向深海大洋的迫切需求有着密切的 关系. 全球海洋预报的突出特点是使用并生成海量的数据, 充分体现了大数据的基本特征. 本 文从论述大数据的起源、概念和本质开始, 介绍了全球海洋预报的基本理论, 进一步结合数据 同化、模式数据和产品分发等 3 个方面具体阐述了全球海洋预报中使用的观测数据和生成的模

关键词 全球海洋预报 科学大数据 海洋数据 海洋观测 式数据等大数据. 最后展望了全球海洋预报以及海洋大数据未来发展中面临的挑战和亟需解决 的关键科学问题.

\section{1 科学大数据概述}

\section{1 大数据起源}

随着信息技术快速发展，互联网和计算机广泛 普及，科学计算、网络交易和社交媒体等应用日益增 多, 音频、视频、文字和图片等数据大量涌现, 数据 存储量、规模、种类飞速增长, 一个薮新的大数据时 代已经到来. 据IDC公司统计, 2011年全球被创建和 被复制的数据总量为 $1.8 \mathrm{ZB}$, 远远超过人类有史以来 所有印刷材料的 $200 \mathrm{~PB}$ 数据总量 ${ }^{[1]}$, 全球数据总量还 以每两年翻一番的速度骤增, 预计到2020年将达到 $40 \mathrm{ZB}^{[2]}$.

学术界、工业界以及政府部门对大数据都表现出 极大的兴趣, 大数据自 2008 年以来得到了快速发展. Nature在2008年首先发表“Big Data”专刊 ${ }^{[3]}$, Econo$m i s t$ 在 2010年刊登“Data, data everywhere”文章 ${ }^{[4]}$, Science 在2011年推出“Dealing with Data”专刊 ${ }^{[5]}$, 麦 肯锡研究院在2011年发表“Big Data: The Next Frontier for Innovation, Competition, and Productivity”报 告 ${ }^{[6]}$, 美国Divyakant Agrawal等20名专家在2012年撰
写发布“Challenges and Opportunities with Big Data” 白皮书 ${ }^{[7]}$. 2012年，美国奥巴马政府宣布投资2亿美元 启动“大数据研究和发展计划”.

\section{2 大数据概念和本质}

现有大数据定义一般从特征提取和归纳总结两 个方面提出, 但目前尚未有一个统一公认的定义. 例 如, 麦肯锡研究院对大数据定义为: 所涉及的数据集 规模已经超过了传统数据库软件获取、存储、管理和 分析的能力; 维基百科给出的大数据定义为: 数据 量规模巨大到无法通过人工，在合理时间内达到截 取、管理、处理并整理成为人类所能解读的信息; IBM 则用 4 个特征相结合来定义大数据: 数量(Volume)、 种类 (Variety)、速度 (Velocity) 和真实 (Veracity $)^{[8]}$; 互 动百科和国家数据公司IDC也提出 4 个特征来定义大 数据, 但与 IBM定义不同的地方, 将第 4 个特征由真 实(Veracity)替换为价值 (Value $)^{[9]}$.

虽然大数据未有明确的定义, 但对大数据存在 以下共识, 即大数据具有数量巨大 (Volume)、种类多 样(Variety)和处理时效紧(Velocity)的 $3 \mathrm{~V}$ 基本特征.

引用格式: 王辉，刘娜，逢仁波，等. 全球海洋预报与科学大数据. 科学通报, 2015, 60: 479-484 Wang H, Liu N, Pang R B, et al. Global ocean forecasting and scientific big data (in Chinese). Chin Sci Bull, 2015, 60: 479-484, doi: $10.1360 / \mathrm{N} 972014-01083$ 
与传统的逻辑推理研究不同, 大数据研究的本质是 对数量巨大的数据做统计性的搜索、比较、聚类和分 类等分析归纳，进行关联性分析，利用数据间的关 系, 找出具有价值的信息 ${ }^{[10]}$. 由于大数据的海量数 据以及处理实时性需求, 对现有的计算机硬件、网络 和数据处理算法提出重大挑战, 需要为之开发性能 和效率更高的大数据基础平台和支撑技术. 因此, 在 大数据时代, 云计算、并行文件系统、新型数据库设 计、快速索引和查询算法等得到了快速发展.

随着科学技术的日益发展, 科学技术相关的数 据日益增多, 与科学相关大数据被称之为科学大数 据. 随着海洋观测和预报技术快速发展, 相关数据量 爆炸式增长, 海洋大数据发展正成为科学大数据的 重要应用之一. 海洋大数据主要包括雷达和卫星等 观测资料、数值预报模式结果以及预报产品等数据. 根据以往关于海洋数据量的研究 ${ }^{[5,11 ~ 13]}$, 估计 2009 2030年全球海洋数据量增长趋势如图 1所示, 2014年全球各种海洋数据总量约为 25 PB, 预计 2030 年全球海洋数据总量将达到 $275 \mathrm{~PB}$. 科学大数据将 复杂性、综合性、全球性和信息与通信技术高度集成 性等诸多特点融于一身, 其研究方法也正在从单一 学科向多学科、跨学科方向转变, 从自然科学向自然 科学与社会科学的充分融合方向过渡 ${ }^{[14]}$.

\section{2 全球海洋预报}

全球海洋预报是采用海洋观测系统收集和处理 的数据作为近实时高质量的输人场, 通过高效的资 料同化系统和数值模式对覆盖全球范围包括两极地 区的多种时空尺度上的海洋状况和海洋现象提供未 来环境演替的预测 ${ }^{[11]}$. 面向复杂的非线性和多时空 尺度海洋过程的海洋模型构建与定量分析, 一方面 需要高性能计算机系统的支撑, 另一方面是对海量 数据存储、快速处理、高效访问和实时分析的需求. 随着高速度、大容量的高性能计算机及存储系统的快 速发展, 加快了全球海洋预报的发展步伐. 国内外许 多国家在科学研究和业务化应用的基础上, 通过完 成全球海洋预报系统模型构建、多尺度表达、数据集 成和可视化等复杂问题, 实现全球海洋系统的模拟 及预报.

国际上对发展全球海洋预报系统高度重视, 在 全球海洋数据同化实验(GODAE)的支持下, 美国和 欧洲为代表的发达国家和地区已经发展常规全球海 洋预报系统，用于发布业务化服务 ${ }^{[15]}$. 现阶段全球 预报系统中有少数的模式(HYCOM/NCODA, Mercator)能够达到全球 $1 / 10^{\circ}$ 分辨率. 大多数系统采用逐级 嵌套加密的方法来达到局部的 $1 / 10^{\circ}$ 分辨率, 如日

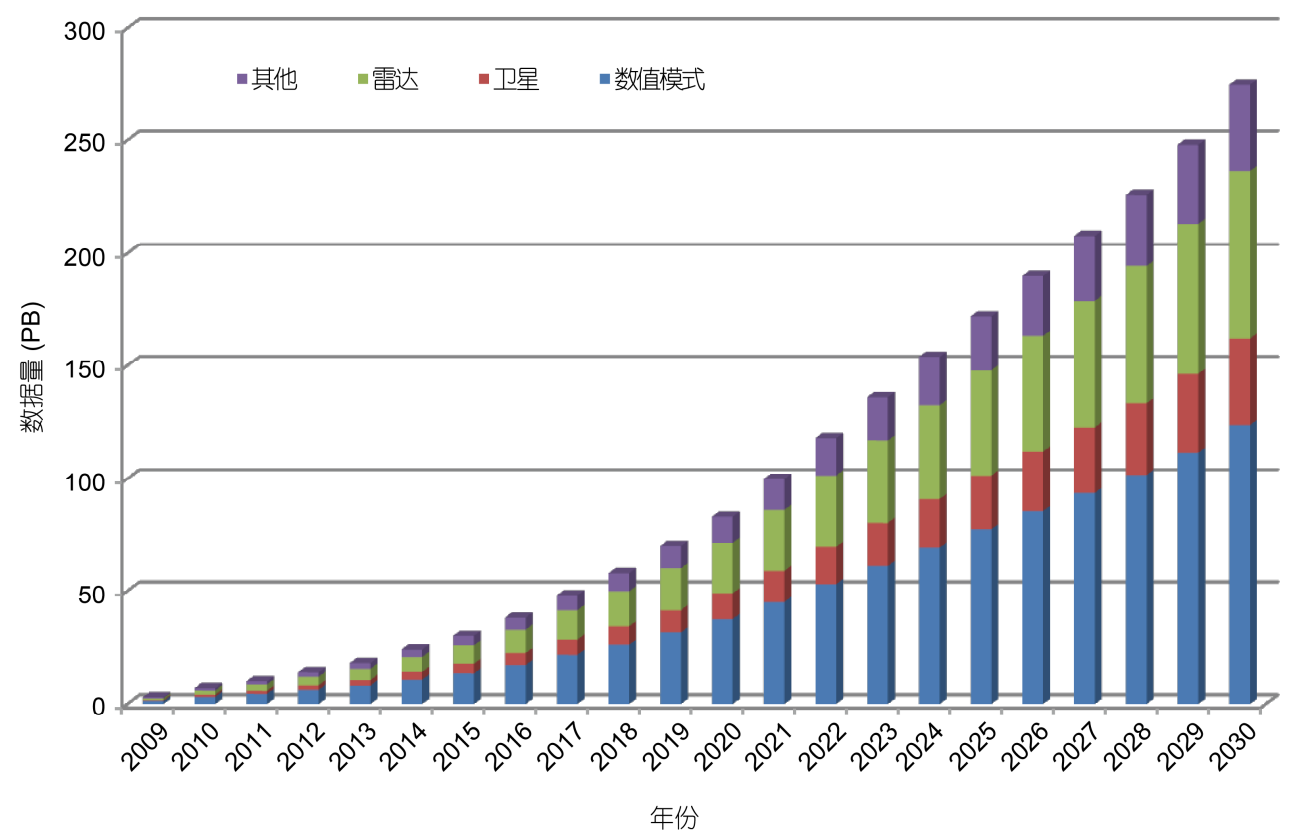

图 1 2009 2030 年全球海洋数据量增长趋势

Figure 1 Increase of the volume of global ocean data in 2009-2030 
本的 Move/MRI系统, 从全球 $1^{\circ}$ 嵌套到北太平洋 $1 / 2^{\circ}$ 再在黑潮区域加密到 $1 / 10^{\circ}$; 澳大利亚的BLUElink系 统, 从全球 $1^{\circ}$ 在澳大利亚周围加密到 $1 / 10^{\circ}$. 全球海 洋预报的实际应用需求促使各国海洋预报机构投人 大量人力和财力提高高性能计算速度和存储能力. 如美国国家海洋和大气管理局与欧洲中期天气预报 中心现有高性能计算系统计算能力分别为 1 亿亿次和 1.5 亿亿次，同时投人巨资建设存储系统，美国国家 海洋和大气管理局以及欧洲中期天气预报中心现有 存储系统均超过 3 PB容量, 并不断持续增加存储系 统容量. Mercator Océan全球预报系统每年的总存储 量达到60 TB. 例如, 美国国家海洋和大气管理局每 日接收 35 亿个观测数据, 生成 150 万个预报产品 ${ }^{[16]}$; 国家海洋环境预报中心每天观测数据和预报产品的 数据量超过 $500 \mathrm{~GB}, 1$ 年数据总量超过 $180 \mathrm{~TB}, 30$ 年 数据总量超过 $5 \mathrm{~PB}$. 近年来, 我国科学家在全球海洋 预报领域做了大量工作，在“十二五”国家科技支撑 计划项目的支持下，国家海洋环境预报中心研发并 建立“全球海洋环境数值预报系统”, 开展了全球海 面风场、全球海浪、海洋环流和极地海冰数值预报关 键技术研究，预报区域由中国海逐渐向全球扩展，建 立起由观测、数据传输、分析、预报、产品制作与分 发等环节构成的我国首个涵盖全球大洋的综合业务 化海洋学预报系统. 该预报系统是包括海冰等预报 要素在内的复杂多界面预报系统, 体现了我国海洋 数值预报技术的发展和进步, 为我国对深海大洋海 洋环境的迫切需求提供有力保障, 具有重要意义. 同 时, 国家海洋环境预报中心积极参与国际业务化海 洋预报组织(GODAE OceanView), 扩大了中国在海 洋预报领域的国际影响.

全球海洋预报关注多变量、多尺度和高度时空属 性的复杂海洋过程的时空要素分析和非线性、多过程 海洋系统的建模以及全方位、多层次的公众服务与决 策支持. 随着海洋预报精度的不断提高, 需要计算的 海域增大、分辨率提高、物理过程参数化描述更为复 杂. 从单一的海洋预报服务走向多源、多变量、异构 和多尺度的海量空间数据的汇聚与分析，提供定时、 定点、定量化的全球海洋数值预报, 通过多层次、多 要素预报产品完整描述海洋结构及其变化. 以全球 海洋数值预报为基础, 多种释用技术和方法的综合 应用, 增加海洋预报服务的时效性、准确性及产品的 多样性, 为深人研究地球系统中全球海洋发展变化
规律提供了重要依据.

\section{3 全球海洋预报与大数据}

在全球海洋预报领域, 海量数据充分体现了体 量巨大、类型多样和要求处理速度快等大数据的基本 特征. 在体量上, 海洋预报相关数据和产品的数据总 量非常庞大; 在类型上, 海洋预报系统所应用的数据 涉及观测资料、预报产品和分析产品等多类数据. 随 着大数据时代的到来, 需要对观测及模拟的海量数 据进行快速、及时地分析和处理，以在较短的时间内 构建准确的、实时的预报数据. 如何利用科学大数据 技术, 结合海洋预报应用特点, 提高海洋大数据查询 和分析效率，提升预报模式的实效性和准确性，成为 海洋大数据发展的关键问题.

全球海洋预报中的数据包括海洋观测数据和海 洋数值模式数据两个部分. 海洋观测系统利用现场 观测和遥感观测等各种观测和探测技术手段，获取 海洋物理、气象等要素数据, 并实现观测数据的无缝 数据传输、海量存储、处理分析以及分发. 经过质量 控制的观测数据在全球海洋预报中一方面可以应用 于数值预报中的模式数据同化, 使系统模拟的海洋 状态尽可能与观测的真实海洋相接近, 另一方面可 用于预报产品的检验和评估. 海洋数值模式数据具 有大空间尺度和高时间分辨率的特征，随着数值预 报精度的提高, 模式数据具有多种类型、丰富信息量 和海量数据等特点. 数值预报产品经由网络专线、卫 星通讯和互联网等方式发给服务保障目标，向公众 提供服务. 全球海洋预报中大数据的处理与分析涉 及数据同化、模式数据和产品分发等 3 个方面. 对全 球海洋预报中这3方面有针对性的加以重点分析和研 究, 将深人理解全球海洋预报中科学大数据的机理 和特点.

全球海洋预报系统将各种不同来源, 不同时空分 辨率的原始观测资料进行反演、订正和分析等处理, 通过同化大量准实时海洋观测资料, 为海洋数值预报 模式提供一个尽可能准确的初始状态，提高海洋预报 的准确度 ${ }^{[17 ~ 20]}$. 海洋观测系统中海量的卫星遥感资 料、浮标等资料为海洋数值模式提供了更大的空间尺 度、更短的更新周期、更丰富的观测信息和内容.

全球海洋预报系统建立了以大规模并行计算为 支撑的较完备的数值预报体系, 需要高性能计算设 备来运行高分辨率的全球海洋预报系统 ${ }^{[21,22]}$. 高性 
能计算速度的快速提高离不开海洋预报等实际应用 需求的发展, 如美国国家海洋和大气管理局计划在 2023年将推出 WoF(Warn-on-Forecast) 系统, 该系统 可为全美和邻近海域提供精细化天气预报和灾害预 警, 美国本土计算网格大小精细至3 10 km, 全球区 域内网格精细至 $15 \mathrm{~km}$, 该系统的计算需求高达 1 万 亿亿次，是目前全球计算速度最快“天河二号超级计 算机系统”计算性能的 182 倍. 研究全球海洋数值预 报模式及模式数据, 在海量的数值预报数据中获得 海洋过程及变化的信息, 需要通过数值模式数据研 究海洋过程的发生、传播和反馈机理, 各种时空尺度 上海洋与大气的相互作用和海气界面过程.

建立以数值预报为支撑, 从数值预报中提取有 用信息, 以统计学、人工智能为基本方法的数值预报 产品解释应用和分发系统 ${ }^{[23]}$. 按照时间、空间和要素 类型等条件进行数据输出, 数值预报产品的展示方 式随着可视化技术的发展经历了从静态到动态，从 二维到三维、四维的进化过程. 在全球业务化海洋预 报系统中实现海量大尺度数值预报产品的时空四维 可视化. 建立以高性能人机交互系统为支撑的预报 制作平台, 更全面地分析和理解海洋运动变化的特 征及其相互间的关系.

\section{4 未来发展趋势}

\section{1 大数据的发展趋势}

在当今高度的信息化社会里, 数据是重要的软 资源, 对社会、科技和经济等的发展起到重要支撑促 进作用. 大数据已经成为了国际社会的研究热点问 题, 在以和平和发展为主题的国际形势下, 谁在大数 据这个战场上占得先机, 谁将掌控国际竞争主动权. 大数据的发展趋势与大数据的特点、技术难点之间存 在必然的联系，其发展就是解决一系列难点问题的 过程. 大数据的复杂性给大数据的研究和应用带来 了巨大挑战, 尤其在数据存储、信息萃取和信息表达 等重要环节尤为突出, 大大增加了数据计算的复杂 度, 传统的数据存储和分析方法几乎无法胜任, 因 此, 揭示、度量、刻画数据复杂性, 并厘清其中的内 在关联机理成为了大数据研究中的首要任务 ${ }^{[24]}$.

大数据对于系统架构, 不管是存储系统、传输系 统还是计算系统等都提出了更高的要求, 现有的数 据中心技术已经难以满足大数据的应用需求. 由于
大数据具有多源、异构和海量等特点, 目前的存储能 力的发展速度远远赶不上数据的增长速度, 必须提 出分布式多源数据的汇聚机制和模型, 如多数据中 心架构、分布式数据仓库以及虚拟数据聚合和数据更 新模型来提高数据获取、组织和使用的效率 ${ }^{[25]}$; 同时 还需要研究高性能、大规模并行的海量数据计算模型 与处理平台来提高海量数据的计算和处理性能, 目 前的云计算技术就是在计算量越来越大、数据越来越 多、越来越动态、越来越实时的需求背景下被催生出 来的一种基础架构. 此外, 在大数据的高速传输、数 据安全等方面也提出了革命性的新技术需求.

\section{2 海洋观测数据的发展趋势}

海洋预报数据是错综复杂大数据的重要组成部 分, 具有大数据的特性. 目前海洋观测系统在获取数 据的时空分辨率、时空覆盖和观测要素类型等方面均 有了较大发展, 但是还不能完全满足海洋环境业务 化预报的发展需求.

面对海洋环境预报行业的实际需求，全球海洋 观测系统建设工作还需要继续发展和完善. 首先, 继 续加强海洋观测能力的建设, 加密海洋观测站, 扩展 浮标观测站网, 进一步提高航空和航天遥感新技术, 并不断拓展海洋观测平台; 其次, 进一步巩固、完善 有线和无线相结合的海洋环境观测数据传输网络, 提高数据传输速度, 提高网络安全保障, 最大程度减 小数据时效性价值的损失, 为满足聚合高效率和高 带宽的数据访问需求, 采用并行 IO通信技术成为必 然趋势; 第三, 完善海洋观测数据的标准和规范, 提 高数据的实用性; 第四, 由于大容量、高性能的存储 在价格方面较高, 推动了存储与数据分离的趋势. 在 分离的基础上，促进基于存储对象技术和层次化存 储技术, 增强存储系统的层次化, 加快在线存储、近 线存储和离线存储三级存储结构发展, 目前三级存 储系统结构已经在国内外海洋预报机构的超级计算机 系统中得到广泛应用; 第五, 建立全球性的海洋观测 资料共享机制, 最大限度发挥观测数据的应用价值.

\section{3 大数据时代下海洋环境预报的发展趋势}

随着数值预报技术的快速发展，预报产品空间 精度不断提高, 预报要素内容逐渐增多, 预报时效不 断提高, 加之数据同化技术的引人, 海洋预报对数据 的吞吐量越来越可观. 海洋环境预报需要对海量数 
据进行快速处理、实现从数据到预报产品的快速转 化, 因此, 为了提高数据获取、组织和使用的效率, 需要配套高性能、大规模、并行的海量数据处理算法 模型和系统平台, 针对海洋大数据多变量、多过程、 非线性和高度耦合的特点, 分析获取本质的信息. 通 过数据质量控制, 降低噪声、消除错误数据, 提高数 据质量. 研究高效率低成本的数据存储方式, 包括多 源数据高质量获取与整合、流式数据的高速索引创建
与存储、错误自动检测与修复以及低质量数据的近似 计算 ${ }^{[10]}$, 降低数据存储成本. 使用海量数据驱动复 杂的海洋动力模型, 提供海洋预报和决策支持. 海洋 大数据的发展需要协调海洋数据源, 共同研究其组 织、存储、传输、分析、可视化方法和技术的一般规 律, 关注海洋大数据的数据关联、空间变换、系统演 化等特征 ${ }^{[26]}$, 进而从机理层面来形成海洋大数据及 其系统的理论和方法.

\section{参考文献}

1 Li G J. Scientific value of big data research (in Chinese). Commun China Comput Federat, 2012, 8: 8-15 [李国杰. 大数据研究的科学价 值. 中国计算机学会通讯, 2012, 8: 8-15]

2 Gantz J, Reinsel D. The digital universe in 2020: Big data, bigger digital shadows, and biggest growth in the Far East. Framingham: IDC Analyze the Future, 2012

3 Sue N. Big data: The Harvard computers. Nature, 2008, 455: 36-37

4 Cukier K. Data, data everywhere: A special report on managing information. Economist, 2010

5 Jonathan O T, Meehl G A, Bony S, et al. Climate data challenges in the 21st Century. Science, 2011, 331: 700-702

6 Manyika J, Chui M, Brown B, et al. Big Data: The Next Frontier for Innovation, Competition, and Productivity. McKinsey Global Institute, 2011. 1-137

7 Agrawal D, Bernstein P, Bertino E, et al. Challenges and opportunities with big data. A Community White Paper Developed by Leading Researchers Across the United States, 2012

8 Meng X F, Ci X. Big data management: Concepts, techniques and challenge (in Chinese). J Comput Res Dev, 2013, 50: 146-169 [孟小峰, 慈祥. 大数据管理：概念、技术与挑战. 计算机研究与发展, 2013, 50: 146-169]

9 Barwick H. The "four Vs" of Big Data. Implementing Information Infrastructure Symposium, 2012

10 Li G J, Cheng X Q. Research status and scientific thinking of big data (in Chinese). Bull Chin Acad Sci, 2012, 27: 647-657 [李国杰, 程 学旗. 大数据研究: 未来科技及经济社会发展的重大战略领域一一大数据的研究现状与科学思考. 中国科学院院刊, 2012, 27: 647-657]

11 Schiller A, Brassington G B. Operational Oceanography in the 21st Century. Netherland: Springer, 2011

12 National Oceanic and Atmospheric Administration. Plan for Reprocessing Large Datasets. 27th IEEE MSST, 2011

13 Starosta D. Data processing, product generation and distribution at the NWS National Centers for Environmental Prediction. FOS Meeting-NCEP Computing, 2012

14 Guo H D, Wang L Z, Chen F, et al. Scientific big data and digital earth (in Chinese). Chin Sci Bull, 2014, 59: 1047-1054 [郭华东, 王力 哲, 陈方, 等. 科学大数据与数字地球. 科学通报, 2014, 59: 1047-1054]

15 Dombrowsky E, Bertino L, Brassington G B, et al. GODAE systems in operation. Oceanogr Mag (Special Issue on the Revolution of Global Ocean Forecasting-GODAE: 10 years of achievement), 2009, 22: 80-95

16 Donoho N, Seybold M, Renkevens T. Operational environmental satellites in the era of Big Data: Progressive report for 2012 and beyond. 93rd American Meteorological Society Annual Meeting, 2013

17 Guan Y H, Zhou G Q, Lu W S, et al. Theory development and application of data assimilation methods (in Chinese). Meteorol Disast Red Res, 2007, 30: 1-8 [官元红, 周广庆, 陆维松, 等. 资料同化方法的理论发展及应用综述. 气象与减灾研究, 2007, 30: 1-8]

$18 \mathrm{Li} \mathrm{H}, \mathrm{Xu}$ J P. Development of data assimilation and its application in ocean science (in Chinese). Mar Sci Bull, 2011, 30: 463-472 [李宏, 许建平. 资料同化技术的发展及其在海洋科学中的应用. 海洋通报, 2011, 30: 463-472]

19 Xiao X J, He N, Zhang Z Q, et al. Variation assimilation using satellite data of sea surface temperature and altimeter (in Chinese). J Trop Oceanogr, 2011，30：1-8 [肖贤俊，何娜，张祖强，等. 卫星遥感海表温度资料和高度计资料的变分同化. 热带海洋学报，2011，30: $1-8]$

20 Xiao X J, Wang D X, Yan C X, et al. Evaluation of a 3DVAR system for the South China Sea. Prog Nat Sci, 2008, 18: 547-554

$21 \mathrm{Pu} \mathrm{Y,} \mathrm{Li} \mathrm{L} \mathrm{J.} \mathrm{The} \mathrm{application} \mathrm{of} \mathrm{thousands} \mathrm{of} \mathrm{CPU} \mathrm{cores} \mathrm{in} \mathrm{high} \mathrm{resolution} \mathrm{Earth} \mathrm{System} \mathrm{Models} \mathrm{(ESM)} \mathrm{(in} \mathrm{Chinese).} \mathrm{E-Sci} \mathrm{Technol} \mathrm{Appl,}$ 2010, 11: 69-75 [普业，李立娟. 高分辨地球系统模式的千核应用. 科研信息化技术与应用, 2010, 11: 69-75] 
22 Wang B. A typical type of high-performance computation: earth system modeling (in Chinese). Physics, 2009, 38: 569-574 [王斌. 一种 典型的高性能计算: 地球系统模拟. 物理, 2009, 38: 569-574]

23 Huang D M, Du Y L, He Q. Migration algorithm for big marine data in hybrid cloud storage (in Chinese). J Comput Res Dev, 2014, 51: 199-205 [黄冬梅, 杜艳玲, 贺琪. 混合云存储中海洋大数据迁移算法的研究. 计算机研究与发展, 2014, 51: 199-205]

24 Wang Y Z, Jin X L, Cheng X Q. Network big data: present and future (in Chinese). Chin J Comput, 2013, 36: 1125-1138 [王元卓, 靳小 龙, 程学旗. 网络大数据: 现状与展望. 计算机学报, 2013, 36: 1125-1138]

25 Zhang Y, Chen M, Liao X F. Big data applications: A survey (in Chinese). J Comput Res Dev, 2013, 50: 216-233 [张引, 陈敏, 廖小飞. 大数据应用的现状与展望. 计算机研究与发展, 2013, 50: 216-233]

26 Zhang Y Q. Promote Big Data to National Strategy (in Chinese). China Economic Report, 2014, 1 [张影强. 将大数据提升为国家战略. 中国经济报告, 2014, 1]

\title{
Global ocean forecasting and scientific big data
}

\author{
WANG Hui, LIU Na, PANG RenBo \& SUN XiaoYu \\ National Marine Environmental Forecasting Center, Beijing 100081, China
}

Global ocean forecasting is one of the future priorities of marine forecasting. This is closely related to the implementation of marine strategy, maintenance of maritime rights, and development of deep sea resources, which are in increasing demand for marine activities across the global ocean. The prominent characteristic of global ocean forecasting is the generation and use of vast amounts of data, which fully reflects the basic characteristics of big data. In this paper, starting from the origin, concept and nature of big data, we define the basic theory of global ocean forecasting. We illustrate big data from observation and models in global ocean forecasting from three aspects, data assimilation, model data, and product distribution. Finally, we discuss key scientific issues in global ocean forecasting and scientific big data in the context of future challenges.

global ocean forecasting, scientific big data, oceanic data, ocean observation

doi: 10.1360/N972014-01083 